\title{
Pinpointing Problems: A Literature Review of Diabetic Peripheral Neuropathy
}

\author{
Nicholas Young ${ }^{1}$ and Savita Khanna ${ }^{2}$ \\ ${ }^{1}$ Indiana University School of Medicine, ${ }^{2}$ Indiana Center for Regenerative Medicine \& \\ Engineering, Department of Surgery, Indiana University School of Medicine
}

A subset of diabetic polyneuropathy, DPN is the most common neurological complication of diabetes. DPN affects around $12-50 \%$ of individuals with diabetes mellitus. DPN compromises the function of nerves in the hands, feet, legs, and arms. It is common for DPN to start in the feet, and often both feet are affected at once. This review of the literature seeks to summarize the current state of DPN research and key findings.

Dissemination of community awareness about the diabetic foot may play a major role in managing DPN. Clinical tests for DPN are simple but lack a standardized approach for the quantification of pain and sensory loss. Thus, estimates of prevalence or intervention efficacy vary widely. Research into burden of disease focuses on ulceration, an ominous sign resulting in amputation and high morbidity/mortality. DPN increases the risk of fall, deteriorates mental health conditions, causes disability lowering quality of life. Specific research regarding the role of social determinants of health in DPN is lacking. It is clear, however, that race, low income, and lack of health insurance are associated with higher burden. Controlling blood glucose is highly effective in managing DPN. It can prevent DPN in Type 1 diabetes and can be effective for preventing DPN in Type 2 diabetes. Treatment of DPN is symptomatic and of limited efficacy in only a fraction of patients, relying primarily on antidepressants and anticonvulsants for the relief of pain. In management of DPN, exercise and psychological therapies can improve outcomes and improve nerve conduction velocity. Combined lifestyle interventions have shown success in prevention of microvascular complications of diabetes and ulceration and may be effective in DPN as well. Harmonization of quantitative diagnostic criteria and definitions of diabetic neuropathies would provide more useful endpoints for trials, epidemiological and mechanistic insight, and potential for earlier detection. 